\title{
ASTHMA
}

\section{Cough frequency in children with mild asthma correlates with sputum neutrophil count}

\author{
A M Li, T W T Tsang, D F Y Chan, H S Lam, H K So, R Y T Sung, T F Fok
}

See end of article for authors' affiliations .....................

Correspondence to: Dr A M Li, Department of Paediatrics, Prince of Wales Hospital, The Chinese University of Hong Kong, Shatin, Hong Kong albertmli@cuhk.edu.hk

Received 1 August 2005 Accepted 3 April 2006 Published Online First 2 May 2006
Background: A study was undertaken to measure cough frequency in children with stable asthma using a validated monitoring device, and to assess the correlation between cough frequency and the degree and type of airway inflammation.

Methods: Thirty six children of median age 11.5 years (interquartile range (IQR) 9-14) with stable asthma were recruited. They underwent spirometric testing, exhaled nitric oxide (eNO) measurement, sputum induction for differential cell count, and ambulatory cough monitoring for 24 hours. Coughing episodes were counted both as individual spikes and as clusters.

Results: All children had mild intermittent asthma and their median forced expiratory volume in 1 second and eNO were 83.3\% (IQR 81.1-97.6) and 56.1 ppb (IQR 37.4-105), respectively. The median number of cough episodes per day was 25.5 (IQR 16-42.8). Sputum induction was successful in $69 \%$ of the subjects and cough frequency was found to have a significant positive correlation with sputum neutrophil count $(r=0.833, p=0.0001)$.

Conclusions: Children with stable mild asthma have increased cough frequency that might be driven by a neutrophilic inflammatory pathway.
C ough is a very common symptom of respiratory diseases. It is audible, can cause sleep disturbance, and may also represent serious underlying disorders. It is therefore unsurprising that parents are frequently anxious about their children's cough and often seek medical advice and remedies. ${ }^{1}$ It has been reported that lack of sleep and choking were the two most common concerns expressed by parents in relation to their children's cough. ${ }^{1}$ Children with asthma can present with cough as the only symptom and, in some cases, cough has been shown to be a dominant feature of asthma exacerbations. ${ }^{2}$ In a study from South Africa, only $2.2 \%$ of children with classical asthma wheeze did not cough. ${ }^{3}$ In an unselected group of children admitted to hospital for an asthma exacerbation, almost $50 \%$ of parents reported that cough was usually or always present. ${ }^{2}$ When cough was objectively measured, nocturnal cough was present in all children recovering from an acute exacerbation and in $39 \%$ of children with stable asthma. ${ }^{45}$ In a study of children with severe asthma, Li et al ${ }^{6}$ reported increased cough frequency compared with normal controls. Whether cough frequency in children with a milder form of asthma is also increased and whether the frequency of cough correlates with underlying airway inflammation is unclear.

There are several difficulties in quantifying clinically relevant cough. Subjective recording of cough by means of diary cards and patients' reports of cough frequency can be very variable and its reliability has been questioned..$^{7-11}$ Conventional tape recorders have been used to quantify cough objectively. ${ }^{8}$ However, tape recorders are bulky and the lack of portability precludes their use during normal daily activities. In addition, they rely only on a single audio signal. The use of an ambulatory cough monitor has recently been validated in both adults and children. ${ }^{12-14}$ The device is highly acceptable to children and no adverse effects have been reported during recording. ${ }^{6}{ }^{13-15}$

In this study we aimed to (1) measure cough frequency in children with stable asthma using a validated objective cough monitoring device, and (2) assess the correlation between measured cough frequency and the degree and type of airway inflammation.

\section{METHODS}

\section{Patient selection}

All children with stable mild asthma attending the Paediatric Chest Clinic of the Prince of Wales Hospital between December 2003 and October 2004 were recruited. The children were aged 7-18 years and all were able to cooperate with the tests. The diagnosis of asthma was made on standard grounds. ${ }^{16}$ We defined stable mild asthmatics as those with (1) no disease exacerbation in the preceding 4 weeks necessitating oral corticosteroids or an increased use of inhaled corticosteroids; (2) use of rescue treatment not more than three times a week; (3) no clinical indication for change in treatment medication; and (4) daytime symptoms less than once a week and nocturnal symptoms less than twice a week. Children who had other concomitant nonasthmatic chronic airway diseases such as bronchiectasis, those who used any prescription or over-the-counter medication that might have affected the course of asthma or its treatment (such as traditional Chinese herbal medicine), and children who were currently involved in any other asthma treatment trial were excluded from the study.

Approval for the study was obtained from the ethics committee of the Chinese University of Hong Kong, and the children's parents or guardians gave written informed consent.

\section{Study design}

This was a prospective observational study. A detailed history was taken and thorough physical examination performed on each child. Weight and height were recorded using standardised equipment. The recruited subjects were required to undergo the following assessments as part of their asthma management.

Abbreviations: eNO, exhaled nitric oxide; $\mathrm{FEV}_{1}$, forced expiratory volume in 1 second; FVC, forced vital capacity; IQR, interquartile range 


\begin{tabular}{|c|c|c|c|}
\hline & Median & IQR 25 & IQR 75 \\
\hline Age (years) & 11.5 & 9.00 & 14.00 \\
\hline Height $(\mathrm{cm})$ & 149.1 & 136.8 & 161.3 \\
\hline Weight (kg) & 36.7 & 30.8 & 47.5 \\
\hline No of cough episodes recorded per day & 25.5 & 16.0 & 42.8 \\
\hline eNO (ppb) & 56.1 & 37.4 & 105.0 \\
\hline$\%$ predicted FEV 1 & $83.3 \%$ & $81.1 \%$ & $97.6 \%$ \\
\hline$\%$ predicted FVC & $84.0 \%$ & $91.1 \%$ & $100.0 \%$ \\
\hline \% Eosinophils & 1.8 & 0.5 & 5.1 \\
\hline \% Neutrophils & 8.3 & 6.1 & 18.3 \\
\hline Severity of asthma score & 0.3 & 0.0 & 2.0 \\
\hline Day time cough & 0.2 & 0.0 & 1.2 \\
\hline Night time cough & 0.2 & 0.0 & 1.8 \\
\hline
\end{tabular}

(1) Asthma severity by visual analogue score: a subjective score from 0 to $10(0=$ asymptomatic, $10=$ severe disabling asthma) was obtained from the subjects. A similar visual analogue score was used to assess patients' daytime and night time cough severity $(0=$ no cough, $10=$ severe disabling cough).

(2) Skin prick test to five groups of aeroallergens (house dust mite, cat and dog dander, cockroaches, grass and tree pollens and mixed moulds) was performed using purified allergen extracts. A child was considered atopic if he/she had at least one skin test result that showed an induration with a diameter at least $3 \mathrm{~mm}$ greater than the saline control.

(3) Measurement of exhaled nitric oxide (eNO) using a chemilumiscence analyser (NOA280i, Sievers Instruments, Boulder, CO, USA) sensitive to NO from $1 \mathrm{ppb}$ to $200 \mathrm{ppm}$ and with a resolution of $1 \mathrm{ppb}$ and an accuracy of $\pm 1 \mathrm{ppb}$, according to the American Thoracic Society guidelines. ${ }^{17}$ The subject was comfortably seated without a nose clip and inhaled NO-free air from a reservoir and subsequently exhaled against a resistor. The flow rate was set at $50 \mathrm{ml} / \mathrm{s}$. This online measurement was taken in triplicate and the average recorded.

(4) Spirometry (Spirolab II, MIR, Italy) using standard techniques measuring forced expiratory volume in l second $\left(\mathrm{FEV}_{1}\right)$ and forced vital capacity (FVC). The best of three efforts was compared with local age and sex matched reference values. ${ }^{18}$

(5) Sputum induction: hypertonic saline $(4.5 \%)$ was used and sputum induction carried out using the standard technique. ${ }^{19} 20$ The detailed methodology has been described elsewhere. ${ }^{6}$ The sputum sample was processed within 4 hours by a technician who was unaware of the clinical data of the subjects. Specimens containing squamous epithelial cells of less than $50 \%$ of the total inflammatory cell number were

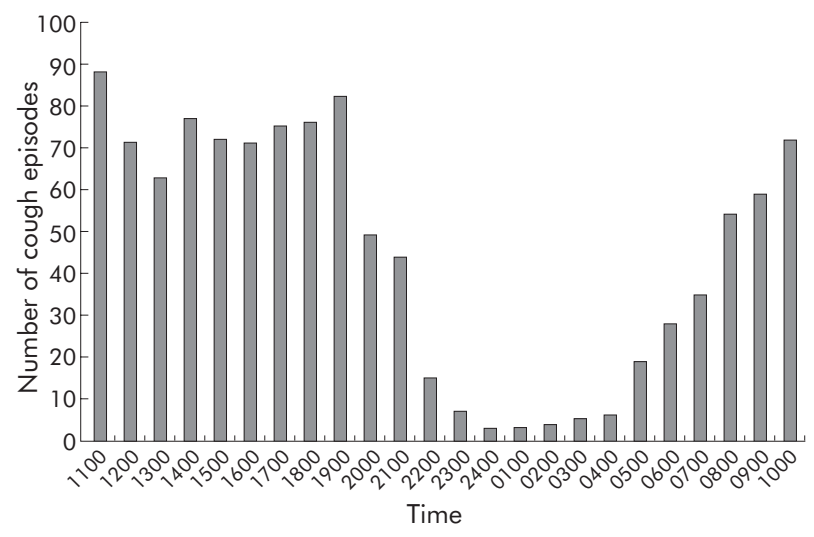

Figure 1 Cough frequency distribution. considered adequate (successful induction). At least 400 inflammatory cells were counted for each specimen. Eosinophil and neutrophil counts were expressed as a percentage of the total cell count.

(6) Cough monitoring: the LR 102 cough recorder used in this study is a modification of the ambulatory cough monitoring device (LR 100) which has been validated for use in children. ${ }^{6}{ }^{14}$ The only difference is that the new device is able to record over a 24 hour period. The LR 102 is a multiparametric recording device, worn in a waist bag, connected to the chest by three electromyographic (EMG) leads and a microphone. Two signals are recorded-a surface EMG and an audio signal. Cough is defined as before by a combination of rapid phasic bursts in both signals and the detailed processing of the recorded signals has been described previously. ${ }^{6}$ Coughing events were counted both as individual spikes and as clusters or bouts. We arbitrarily defined each cluster (a cough epoch) as a close succession of coughs ( $<2$ seconds between individual coughs) recorded by each trigger of the recorder. The cough data were expressed as the total number of cough episodes (individual spike + cough cluster) per unit recording time. The subjects had the monitor put on at 16.00 hours on the study day and were asked to return at the same time the next day for its removal when they performed sputum induction.

\section{Statistical analysis}

The data are presented as medians with interquartile ranges (IQR). Spearman's rank correlation coefficient was used to assess the association between the various parameters. SPSS for Windows statistical software (Release 11.0, SPSS Inc, Chicago, IL, USA) was used in the analyses. The level of significance was set at $5 \%$ in all comparisons, and all statistical tests were two sided.

Table 2 Correlation between cough and other parameters

\begin{tabular}{lll}
\hline & $\begin{array}{l}\text { Correlation } \\
\text { coefficient }\end{array}$ & p value \\
\hline eNO & -0.185 & 0.279 \\
FEV 1 & -0.215 & 0.208 \\
FVC & -0.119 & 0.491 \\
\% Eosinophils & 0.02 & 0.924 \\
\% Neutrophils & 0.833 & 0.0001 \\
Severity of asthma score & -0.077 & 0.679 \\
Self-reported daytime cough & -0.090 & 0.630 \\
Self-reported night time cough & -0.068 & 0.717 \\
\hline
\end{tabular}

eNO, exhaled nitric oxide; $\mathrm{FEV}_{1}$, forced expiratory volume in 1 second; FVC, forced vital capacity. 


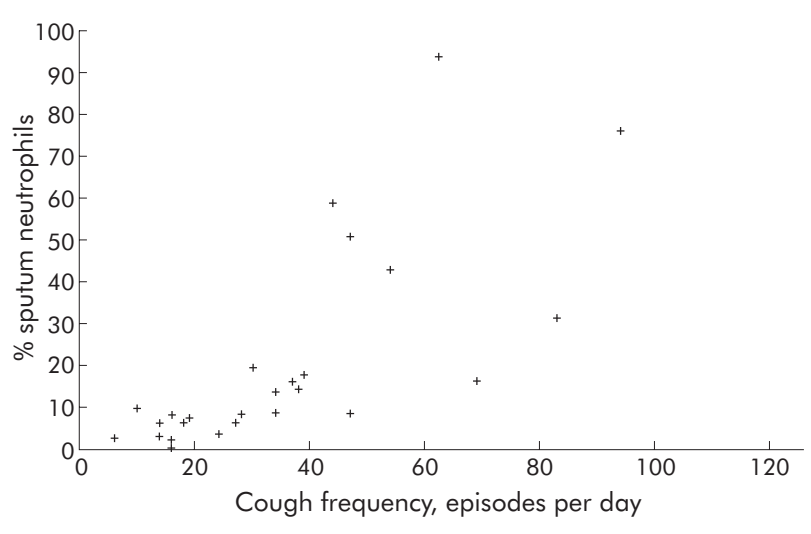

Figure 2 Scatterplot showing the relationship between cough frequency and sputum neutrophils.

\section{RESULTS}

Thirty six children aged $7-17$ years (median age 11.5 years, IQR 9-14) were recruited to the study. There were 24 boys and 12 girls. All subjects were, by definition, atopic. Eleven (31\%) had sensitivity to more than one allergen and all were sensitive to house dust mite. Five patients were using inhaled corticosteroids and the range of beclomethasone equivalent dosage was 200-400 $\mu \mathrm{g}$ (assuming budesonide is equally as potent as beclomethasone and fluticasone is twice as potent). The remaining 31 were using short acting $\beta$ agonists on an "as required" basis. All subjects were only symptomatic with respiratory tract infections and none complained of interval symptoms of asthma-for example, wheeze with weather change. Twenty four subjects had normal spirometric values, 10 had mild obstructive deficit with $\mathrm{FEV}_{1}>70 \%$ predicted, and the remaining two had severe obstructive deficits $\left(\mathrm{FEV}_{1} 46 \%\right.$ and $49 \%$ predicted). Nonetheless, they all considered their asthma to be mild and the median asthma severity score was 0.3. In addition, they did not consider cough to be a significant symptom at the time of the study: the median daytime and night time cough scores were both reported as 0.2. Seven children had passive tobacco smoke exposure. The baseline characteristics of the subjects are summarised in table 1 .

Cough recording was successful in all children and no adverse events or sleep disturbance were reported. The median number of cough episodes per day was 25.5 (IQR 16-43). The distribution of cough episodes over the whole recording period is shown in fig 1 . We were not able to measure sleep stage, so we asked all parents to report the time when the patients slept and woke in the morning. We found that all subjects went to sleep between 22.00 and 24.00 hours and woke between 07.00 and 09.00 hours. We therefore arbitrarily defined night cough as cough occurring between 23.00 hours and 07.00 hours, and daytime cough as cough occurring at any other time. The median number of daytime coughs/hour was significantly greater than night time coughs (71.5/hour (IQR 56.50-76.25) v 6.5/hour (IQR 3.75-29.75), $\mathrm{p}<0.001)$.

Twenty five of the 36 children $(69.4 \%)$ were able to produce an adequate sputum sample on induction. The median eosinophil and neutrophil counts were $1.8 \%$ and $8.3 \%$, respectively. Using Spearman's correlation, cough frequency was found to have a significant positive correlation with the sputum neutrophil count. The results are summarised in table 2 and a scatterplot showing the relationship between cough frequency and sputum neutrophils is shown in fig 2 .

\section{DISCUSSION}

In this study our aims were to assess the cough frequency in a group of children with mild asthma and to examine the relationship between measured cough frequency and airway inflammation. We were able to show that, despite recruiting at a time of apparent stability, our cohort of mild asthmatics had increased cough frequency compared with that reported for normal white children. ${ }^{13}$ Traditional teaching has led us to believe that children with asthma cough more than normal subjects only during exacerbations but not during remission. ${ }^{21}$ This is in sharp contrast to the findings of our current study where children with stable asthma had increased cough frequency. Munyard et $a l^{13}$ used a similar monitoring device on 44 normal white children aged 8-12 years and found a median cough frequency of 11 episodes per day. Our cohort of mild asthmatics had a median cough frequency of 25.5 episodes over a 24 hour period. This increased cough frequency was recorded at a time when the patients were in remission. Furthermore, they did not consider cough to be much of a troublesome symptom at the time of the study as the median cough severity score was only 0.2 . Increased cough frequency has also been reported for stable severe asthmatics with a median cough frequency of 19 episodes per day. ${ }^{6}$ In addition, we found that cough frequency is greater during the day than at night, despite conventional teaching on the importance of nocturnal cough in asthma.

We did not find any correlation between the subjective selfreported daytime cough score $(\mathrm{p}=0.630)$ and night time cough score $(p=0.717)$ with the actual cough frequency recorded. This finding is similar to what has been reported in the literature, questioning the reliability of subjectively reported cough intensity and severity. ${ }^{15-11}$ Although subjective evaluation of patient perception of cough is the most convenient and commonly used tool, it remains an unvalidated and apparently unreliable measure. In addition, we do not know whether the cough severity scale is linear and how much the reported symptoms are affected by the patient's mental state.

Persistent airway inflammation in stable asthmatics may explain our finding of increased total cough. A recent study showed that persistent airway inflammation was still evident in asymptomatic asthmatics with normal lung function. ${ }^{22}$ Ongoing airway inflammation in our group of stable asthmatics is supported by the finding that the median sputum eosinophil count was $1.8 \%$ compared with $0.3 \%$ reported for normal controls. ${ }^{23}$ Chang et al ${ }^{24}$ reported that an increase in cough and eosinophilic inflammation were characteristics of mild asthma exacerbation and Li et al, ${ }^{6}$ In their study of 32 children with stable severe asthma, found a positive correlation between cough frequency and eNO. The patients in our study are, however, very different from those in the above two studies. Our patients were in remission and their asthma had been in a stable state for at least 4 weeks before the study. They were all mild asthmatics and only became symptomatic with respiratory tract infections. Many of them were using only inhaled $\beta$ agonists on an infrequent basis. The failure to demonstrate a positive correlation between measured cough frequency and eosinophilic inflammation as quantified by percentage sputum eosinophil and eNO may be related to the small sample size. On the other hand, our result could raise the question of whether cough is a reliable marker of the degree of eosinophilic inflammation in mild asthmatics during the non-acute state. Interestingly, we found a significant correlation between increased cough and percentage sputum neutrophil count. We did not find any direct relationship between increased cough or percentage sputum neutrophil count with tobacco smoke exposure or environmental pollution. The daily pollution index measured a week before the subjects underwent assessment and on the day of the study did not differ. Besides, none of the subjects complained of a change in their cough frequency during the study period. In children with asthma, neutrophilic inflammation is classically seen during acute 
severe exacerbations. ${ }^{25}$ Whether the detected increased cough actually heralds the onset of an asthma exacerbation cannot be answered by this study as measurements were performed only at a single time point. In adults, neutrophilic inflammation typically occurs in the more severe form of asthma and reflects an infective aetiology. ${ }^{26}{ }^{27}$ We did not look for a possible infective cause for the increased cough found in our cohort of patients, although none of them complained of any symptoms suggestive of a respiratory infection before or during the study. However, we are not able to exclude the possibility that a subclinical ongoing infection was responsible for the increased cough frequency. Nonetheless, there is accumulating evidence to suggest that neutrophilic inflammation is involved in enhanced bronchial reactivity and exacerbation of asthma. ${ }^{28} 29$ The importance of neutrophils as the dominant inflammatory cell in more severe phenotypes of asthma has also been well established in adults. ${ }^{30}$ There is evidence to suggest that children with mild intermittent asthma do not have classical eosinophilic airway inflammation as seen in those with more severe disease. ${ }^{31}{ }^{32}$ Stevenson et $a l^{31}$ reported low or absent eosinophil counts in a group of children with symptoms only precipitated by viral infections and with predominantly mild disease, in many ways similar to our population. Fitch $e a^{32}$ showed that, in children with cough as a prominent symptom, there appeared to be a significant increase in neutrophil count in bronchoalveolar material compared with other symptomatic and control populations. In this study we have provided further evidence that neutrophilic rather than eosinophilic inflammation might be more important in driving ongoing symptoms in children with mild intermittent asthma.

This study has several limitations. Firstly, we recruited only 36 patients. Whether an increased sample size would have shown a significant correlation between cough frequency and other parameters is not known. We did not have normal Chinese children to serve as a comparison to our cohort of patients, but it was not the intention of this study to compare cough frequency between asthmatics and normal children. Using data from a previous study that included a white population ${ }^{13}$ may not be appropriate as environmental and racial characteristics of the two cohorts could have accounted for their differences in cough frequency. Secondly, this study was a cross sectional study at a specific time point. We did not attempt to assess whether the current medications taken by the patients were optimal or not. Longitudinal studies to determine whether increasing asthma treatment or the use of anti-neutrophilic measures (such as antibiotics) are useful strategies to treat increased cough should be carried out and would provide important insight into the pathophysiology of cough in asthma. The main strength of this study, however, was the use of an objective cough monitor relying on two signals to confirm genuine coughing. Sound quality may be affected by the sleep position of the patient and the actual recordings may be contaminated by sounds made by people nearby. Therefore, the use of two signal modalities to confirm an episode of cough greatly improved the reliability and accuracy of the recording.

In summary, we were able to show increased cough frequency in children with mild asthma despite their disease being in remission. The increased cough might be driven by a neutrophilic inflammatory pathway, the significance of which will need to be elucidated in further studies.

\section{Authors' affiliations}

A M Li, T W T Tsang, D F Y Chan, H S Lam, H K So, R Y T Sung, T F Fok, Department of Paediatrics, Prince of Wales Hospital, The Chinese University of Hong Kong, Shatin, Hong Kong
This work was supported by a Direct Grant for Research from the Chinese University of Hong Kong (project code 2041067).

Competing interests: none declared.

\section{REFERENCES}

1 Chang AB. State of the art: cough, cough receptors and asthma in children. Pediatr Pulmonol 1999;28:59-70.

2 Chang AB, Phelan PD, Robertson CF. Cough receptor sensitivity in children with acute and non-acute asthma. Thorax 1997:52:770-4.

3 Green R, Luyt D. Clinical characteristics of childhood asthmatics in Johannesburg. S Afr Med J 1997;87:878-82.

4 Brooke AM, Lambert PC, Burton PR, et al. Night cough in a population-based sample of children: characteristics, relation to symptoms and associations with measures of asthma severity. Eur Respir J 1996;9:65-71.

5 Hoskyns EW, Beardsmore CS, Simpson H. Chronic night cough and asthma severity in children with stable asthma. Eur J Pediatr 1995;154:320-5.

6 Li AM, Lex C, Zacharasiewicz A, et al. Cough frequency in children with stable asthma: correlation with lung function, exhaled nitric oxide, and sputum eosinophil count. Thorax 2003;58:974-8.

7 Archer LNJ, Simpson H. Night cough counts and diary card scores in asthma. Arch Dis Child 1985;60:473-4.

8 Falconer A, Oldman C, Helms P. Poor agreement between reported and recorded nocturnal cough in asthma. Pediatr Pulmonol 1993;15:209-11

9 Brunekreef B, Groot B, Rijcken B, et al. Reproducibility of childhood respiratory symptom questions. Eur Respir J 1992;5:930-5

10 Juniper EF, Guyatt GH, Dolovich J. Assessment of quality of life in adolescents with allergic rhinoconjunctivitis: development and testing of a questionnaire for clinical trials. J Allergy Clin Immunol 1994;93:413-23.

11 Thompson AH, Pratt C, Simpson H. Nocturnal cough in asthma. Arch Dis Child 1987:62:1001-4.

12 Munyard P, Busst C, Logan-Sinclair R, et al. A new device for ambulatory cough recording. Pediatr Pulmonol 1994;18:178-86.

13 Munyard P, Bush A. How much coughing is normal? Arch Dis Child 1996:74:531-4.

14 Hamutcu R, Francis J, Karakoc F, et al. Objective monitoring of cough in children with cystic fibrosis. Pediatr Pulmonol 2002;34:331-5.

15 Corrigan DL, Paton JY. Pilot study of objective cough monitoring in infants. Pediatr Pulmonol 2003;35:350-7.

16 Warner JO. Asthma: a follow up statement from an international paediatric asthma consensus group. Arch Dis Child 1992;67:240-8.

17 American Thoracic Society. Recommendation for standardized procedures for the on-line and off-line measurement of exhaled lower respiratory nitric oxide and nasal nitric oxide in adults and children-1999. Am J Respir Crit Care Med 1999;160:2104-17.

18 Ip MS, Karlberg EM, Karlberg JP, et al. Lung function reference values in Chinese children and adolescents in Hong Kong. Am J Respir Crit Care Med 2000;162:424-9.

19 Smith CM, Anderson SD. Inhalational challenge using hypertonic saline in asthmatic subjects: a comparison with responses to hyperpnoea, metacholine and water. Eur Respir J 1990;3:144-51.

20 Fahy JV, Boushey HA, Lazarus SC, et al. Safety and reproducibility of sputum induction in asthmatic subjects in a multicenter study. Am J Respir Crit Care Med 2001;163:1470-5.

21 Rietveld S, Rijssenbeek-Nouwens LH. Diagnostics of spontaneous cough in childhood asthma: results of continuous tracheal sound recording in the homes of children. Chest 1998:113:50-4.

22 van den Toorn LM, Overbeek SE, de Jongste JC, et al. Airway inflammation is present during clinical remission of atopic asthma. Am J Respir Crit Care Med 2001;164:2107-13.

23 Cai Y, Carty K, Henry RL, et al. Persistence of sputum eosinophilia in children with controlled asthma when compared with healthy children. Eur Respir J 1998;11:848-53.

24 Chang AB, Harrhy VA, Simpson J, et al. Cough, air inflammation, and mild asthma exacerbation. Arch Dis Child 2002;86:270-5.

25 Norzila MZ, Fakes K, Henry RL, et al. Interleukin-8 secretion and neutrophil recruitment accompanies induced sputum eosinophil activation in children with acute asthma. Am J Respir Crit Care Med 2000;161:769-74.

26 Sur S, Crotty TB, Kephart GM, et al. Sudden-onset fatal asthma. A distinct entity with few eosinophils and relatively more neutrophils in the airway submucosa? Am Rev Respir Dis 1993;148:713-9.

27 Pizzichini MM, Pizzichini E, Efthimiadis A, et al. Asthma and natural colds. Inflammatory indices in induced sputum: a feasibility study, Am J Respir Crit Care Med 1998:158:1178-84.

28 Douwes J, Gibson P, Pekkanen J, et al. Non-eosinophilic asthma: importance and possible mechanisms. Thorax 2002;57:643-8.

29 Yasui K, Kanda $\mathrm{H}$, Iwanami T, et al. Increased serum concentration of urinary trypsin inhibitor with asthma exacerbation. Eur Respir J 2003;22:739-42.

30 Kamath AV, Pavord ID, Ruparelia PR, et al. Is the neutrophil the key effector cell in severe asthma? Thorax 2005;60:529-30.

31 Stevenson EC, Turner G, Heaney LG, et al. Bronchoalveolar lavage findings suggest two different forms of childhood asthma. Clin Exp Allergy 1997:27:1027-35.

32 Fitch PS, Brown V, Schock BC, et al. Chronic cough in children: bronchoalveolar lavage findings. Eur Respir J 2000;16:1109-14. 